See Article page 1776.

\section{Commentary: Is aortic septotomy the "Holy Grail" for thoracic endovascular aortic repair management of chronic type B dissection?}

\author{
Michael Troncone, MD, and François Dagenais, MD
}

The article from Fukuhara and colleagues ${ }^{1}$ in this edition of the Journal provides compelling insight in the contemporary management of chronic type $\mathrm{B}$ dissection (cTBAD). The authors retrospectively analyzed 12 years of endovascular interventions for cTBAD, comparing the outcomes between patients receiving thoracic endovascular aortic repair (TEVAR) alone versus TEVAR and endovascular landing zone optimization (ELZO), whether it be proximal or distal. When one assesses the cohorts of patients stratified to each group, a few differences are notable. The follow-up period for the aortic septotomy cohort was 1.3 years, whereas the nonseptotomy cohort was 4 years-a statistically significant difference that would clearly favor the septotomy group for survival as well as freedom composite end points (aortic reintervention, aorta-related death, and/or sudden death). In fact, while a pronounced difference was noted at 8 years, there was no statistical difference in survival or composite end points at 2 years. This was driven primarily by 12 sudden deaths beyond 2 years and a greater rate of aortic reinterventions in the non-septotomy cohort. Of the reinterventions, $77 \%$ were required due to distal retrograde false lumen flow. This is not unexpected with a patent distal re-entry tear, as evidenced by the bleak

From the Department of Cardiac Surgery, Institut Universitaire de Cardiologie et de Pneumologie de Québec, Université Laval, Québec, Québec, Canada.

Disclosures: Dr Dagenais is consultant and proctor for Cook Medical, Edwards Lifesciences, and Medtronic, Inc. Dr Troncone reported no conflicts of interest.

The Journal policy requires editors and reviewers to disclose conflicts of interest and to decline handling or reviewing manuscripts for which they may have a conflict of interest. The editors and reviewers of this article have no conflicts of interest.

Received for publication Aug 12, 2021; revisions received Aug 12, 2021; accepted for publication Aug 13, 2021; available ahead of print Aug 20, 2021.

Address for reprints: François Dagenais, MD, Institut Universitaire de Cardiologie et de Pneumologie de Québec, 2725 Chemin Sainte-Foy, Québec, Québec, Canada G1V 4G5 (E-mail: francois.dagenais@chg.ulaval.ca).

J Thorac Cardiovasc Surg 2023;165:1778-9

0022-5223/\$36.00

Copyright $₫ 2021$ Published by Elsevier Inc. on behalf of The American Association for Thoracic Surgery

https://doi.org/10.1016/j.jtcvs.2021.08.031
Check for updates

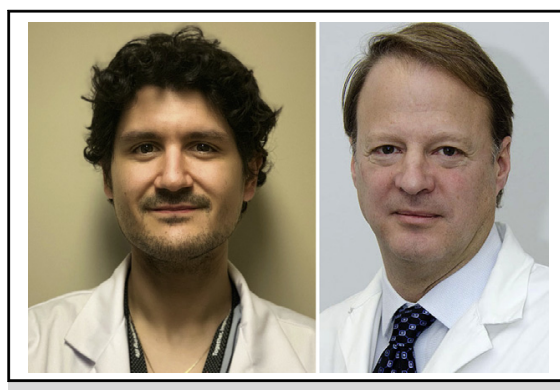

Michael Troncone, MD, and François Dagenais, MD

CENTRAL MESSAGE

Aortic septotomy as an adjunct

to TEVAR for chronic type B

dissection may decrease late re-

intervention and increase posi-

tive aortic remodeling.

literature of isolated TEVAR for cTBAD treatment. As stated by the authors, it is fundamental to detect differences in outcomes between the septotomy and non-septotomy groups over equal, longer follow-up periods.

The authors do point toward improved aortic remodeling in the septotomy cohort as a marker of therapeutic benefit, citing the recent Society for Vascular Surgery/Society of Thoracic Surgeons guidelines ${ }^{2}$ for the reporting standards of type B dissections. In strict accordance with the guideline definitions, positive aortic remodeling did occur. However, it is unclear whether endovascular manipulation of the septum, obligatorily changing aortic luminal geometries, is equivalent to the aortic remodeling purported after reducing false lumen flow with tear coverage. The cited guidelines, published in 2020, are validated for characterizing aortic remodeling after TEVAR, with no mention of adjunctive septotomy.

Previous publications have demonstrated the disparate natural histories of de novo type $\mathrm{B}$ aortic dissections and residual dissections post-type A repair (rTBAD). ${ }^{3}$ In this cohort of 88 patients undergoing treatment for cTBAD, 16 of 31 patients receiving septotomy and 36 of 57 patients not receiving septotomy had rTBAD, most commonly after hemiarch repairs. Of the septotomy group, only 3 required proximal ELZO; all 31 required distal ELZO. Furthermore, since the 36 patients not receiving septotomy with rTBAD received TEVAR, one must assume they similarly had adequate proximal landing zones. This emphasizes the crucial importance of closure of the proximal entry tear and avoidance of DANE (ie, distal aortic new entry tear) 
at the initial type A repair to secure an adequate proximal landing zone for future endovascular procedures.

Procedurally, technical success rate was high, with minimal complications, emphasizing the importance of a dedicated multidisciplinary team for these complex endovascular approaches. There was one intimointimal intussusception during "cheese-wire" septotomy. When reviewing the authors' technique, there is no consensus on the optimal method of "cheese-wire" fenestration, with either direct "pull-down," or "see-saw-ing" the wire. Both have been purported to reduce the incidence of intimointimal intussusception.

The strength of this paper is 3-fold. First, the authors address an extremely complex disease in a manner that does not require advanced devices that obligatorily cover more aortic surface area or the introduction of occlusion devices. This has widespread applicability in anatomically suitable cases. Second, the difference in aortic reintervention is noteworthy. While it is expected that patients without septotomy would require reintervention, the low rate in the septotomy cohort may indicate a durable repair. This could be more borne out with longer follow-up. Third, it highlights one of the principles of surgery for type A repairclosure of the proximal entry tear and avoidance of DANE to ensure a secure proximal landing zone for future interventions. The Michigan group has to be congratulated for an innovative approach in the complex management of patients with cTBAD.

\section{References}

1. Fukuhara S, Khaja MS, Williams DM, Marko X, Yang B, Patel HJ, et al. Aortic Symposium 2021: aortic septotomy to optimize landing zones during thoracic endovascular aortic repair for chronic type B aortic dissection. J Thorac Cardiovasc Surg. 2021;165:1776-86.

2. Lombardi JV, Hughes GC, Appoo JJ, Bavaria JE, Beck AW, Cambria RP, et al. Society for Vascular Surgery (SVS) and Society of Thoracic Surgeons (STS) reporting standards for type B aortic dissections. Ann Thorac Surg. 2020;109:959-81.

3. Krähenbühl E, Maksimovic S, Sodeck G, Reineke D, Schoenhoff F, Schmidli J, et al. What makes the difference between the natural course of a remaining type B dissection after type A repair and a primary type B aortic dissection? Eur J Cardiothorac Surg. 2012;41:e110-5; discussion e115-6. 